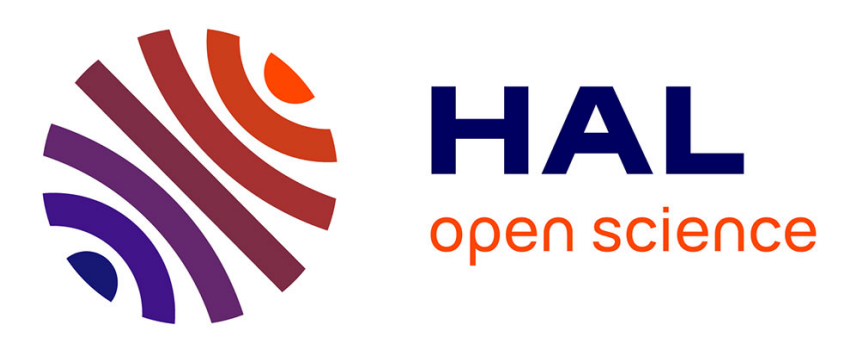

\title{
Influence of the depth and morphology of real cracks on diffuse ultrasound in concrete: a simulation study
}

\author{
Audrey Quiviger, Alexandre Girard, Cedric Payan, Jean François Chaix, \\ Vincent Garnier, Jean Salin
}

\section{- To cite this version:}

Audrey Quiviger, Alexandre Girard, Cedric Payan, Jean François Chaix, Vincent Garnier, et al.. Influence of the depth and morphology of real cracks on diffuse ultrasound in concrete: a simulation study. NDT \& E International, 2013, 60, pp.11. hal-00861445

\section{HAL Id: hal-00861445 \\ https://hal.science/hal-00861445}

Submitted on 12 Sep 2013

HAL is a multi-disciplinary open access archive for the deposit and dissemination of scientific research documents, whether they are published or not. The documents may come from teaching and research institutions in France or abroad, or from public or private research centers.
L'archive ouverte pluridisciplinaire HAL, est destinée au dépôt et à la diffusion de documents scientifiques de niveau recherche, publiés ou non, émanant des établissements d'enseignement et de recherche français ou étrangers, des laboratoires publics ou privés. 


\title{
Influence of the depth and morphology of real cracks on diffuse ultrasound in concrete: a simulation study
}

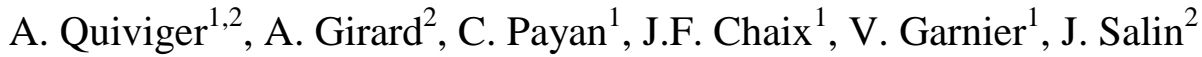 \\ ${ }^{1}$ Aix Marseille Univ., LMA CNRS UPR 7051, LCND IUT Aix en Provence, 13100 \\ Aix en Provence (France) \\ ${ }^{2}$ EDF-R\&D-STEP, 6 quai Watier, BP 49, 78401 Chatou, France \\ Corresponding author: Cedric Payan, Aix Marseille Univ., LMA CNRS UPR 7051, \\ LCND IUT Aix en Provence, 13100 Aix en Provence (France) - \\ cedric.payan@univ-amu.fr - Phone : +33442939055
}

\begin{abstract}
:
The aim of the present paper is to simulate the propagation of diffuse ultrasonic energy in concrete in the presence of a real crack. The numerical model is presented and validated by the comparison with experimental data from the literature. Unlike most of the studies which consider a crack as a notch, a realistic crack morphology exhibits partial contacts along its lips. These contacts are modeled in order to study their influence on the diffusion parameters. The feasibility of determining the contact density of the crack is shown, revealing practice implications for non-destructive crack sizing and imaging in concrete.
\end{abstract}

Keywords: Diffuse ultrasound, numerical simulation, crack, concrete.

Journal: NDT\&E International 


\section{Introduction}

Cracking is critical for the structural integrity of civil engineering structures, as a consequence of the risks of mechanical failure and loss of impermeability which it can produce. In particular, the latter effect is induced by cracking of the concrete cover located between the surface and the first layer of rebar. This leads to the penetration of aggressive agents into the core of the structure, thus promoting corrosion of the rebar, leading to a degradation of the mechanical properties of the structure. The detection and characterization of such cracks is thus necessary, to allow the remaining lifetime of the structure to be predicted and its maintenance to be optimized.

Several difficulties are encountered in the use of acoustic techniques for the characterization of concrete in an industrial context. ISO and ASTM standards [1,2] state that ultrasound frequencies in the range between $20 \mathrm{kHz}$ and $150 \mathrm{kHz}$ should be used. The wavelength is thus greater than $3 \mathrm{~cm}$, which does not allow real cracks to be detected efficiently before reaching the rebar located at few centimeter depth. When the frequency is increased, ultrasound measurements become more complex, in particular due to multiple scattering, resulting from the presence of aggregates similar in size to the ultrasound wavelength [3,4]. Various authors [5-7] have studied the ultrasonic characterization of cracks in concrete. They revealed a change in the waves' time of flight in the presence of a crack with controlled dimensions: a notch, whose walls have no contact points. Analogous results were observed with cracks produced by bending loads [8]. However, these cracks were opened artificially by applying a bending force to the test specimens, thus placing limitations on the accuracy with which they can represent real cracks.

A second approach [9], based on the analysis of diffuse ultrasound, was also studied for the purposes of concrete characterization. It shows that the complex propagation of multiple scattered waves in concrete can be simplified into a standard diffusion law. It is founded on the analysis of the ultrasonic energy diffusion by two parameters: the diffusivity $\mathrm{D}$ (with dimensions $[\mathrm{m}]^{2}[\mathrm{~s}]^{-1}$ ), characteristic of the material's structure, and the dissipation $\sigma$ (with dimensions $[\mathrm{s}]^{-1}$ ), which reflects the medium's viscoelastic properties. Anugonda et al. [9] demonstrated the validity of this approach for concrete, both analytically and experimentally. They thus opened up numerous possibilities for the non-destructive characterization of microstructural damage in concrete. Becker et al. [10] thus studied the variation of diffusion parameters as a function of the aggregate diameters, whereas Punurai et al. [11] determined such variations in cement as a function of the quantity of occluded air. Deroo et al. [12] studied the influence of Alcali Silicate Reaction on the diffusion parameters. Diffuse ultrasound was also analyzed in order to characterize cracks in concrete by Ramamoorthy et al. [13]. They showed that the diffusion parameters vary as a function of the length of a notch in concrete. Authors introduced another parameter: the Arrival Time of Maximum Energy (ATME). Quiviger et al. [14] confirmed the ability of ATME to characterize the opened portion of a real crack. According to the same authors, the ATME also varies as a function of the length of the closed portion of the crack. The presence of partial contacts along the length of the crack is assumed to be the cause of the observed variations.

The aim of the present study is to verify the influence of real crack morphology on the measured diffusion parameters. The morphology of a real crack in concrete and the numerical model are presented. This model is validated by the comparison with experimental data from concrete samples containing controlled notches. A real crack is then introduced by the study of the influence of partial contacts on diffuse ultrasound. 
Then, the numerical model is applied to the case of real cracks in concrete and compared to experimental data from the literature [14].

\section{Crack morphology}

The morphology of a crack, which is open at the concrete surface, has been described in [14]. The crack comprises two portions: the first is the open portion, located at the surface at the outer end of the crack. The second, in which the two walls are partially or totally in contact, is considered to be closed. The particularity of a real closed crack lies in the number and nature of the contact points or areas present between the walls. These modify the mechanical behaviour of the crack, in particular as a result of local stress redistribution.

Turatsinze et al [15] revealed a disparity in the profile of a crack produced by bending, similarly to the test specimens shown in Fig. 1(a) and Fig. 1(b) used in the present study. The crack exhibits a variable morphology, depending on its depth in the sample. These authors revealed the presence of discontinuities in the profile of the crack, lying in the median plane [Fig. 1(c) and Fig. 1(d)]. This is not a simple crack, but it can be represented in this zone by an interfacial crack with contact zones and interlocking effects.

Diffuse ultrasound in a concrete sample was numerically modeled by Ramamoorthy et al. [13] who studied the influence of a numerical notch on ATME. Seher et al. [16] numerically modeled diffusion in the presence of partially closed cracks. However, this study was performed using a commercial heat transfer software, thus the dissipation was taken into account by a post processing procedure (exponential decay). In the following, the numerical model including dissipation is presented and a realistic numerical crack is introduced.

\section{Numerical simulation}

III. 1 The model

The computer code developed in the present study is based on a $2 \mathrm{D}$ finite difference method. This method is chosen mainly as a result of the simple geometry of the problem, the functional regularity of the energy, and the uniform diffusion which can be achieved inside the material. The results given by the numerical simulations are then compared with the experiments carried out by Quiviger et al [14], with the decision to use a $2 \mathrm{D}$ model motivated by the symmetry of the test specimens used in the latter study, as well as by the resulting gain in computing time. The code is developed using Matlab®.

The concrete in which diffuse ultrasound propagates has fixed dissipation and diffusivity values. The size, the position of the sensors and the boundary conditions of the model are also fixed, to ensure that they match the experimental conditions. A rectangular mesh, with a horizontal resolution of $2 \mathrm{~mm}$ and a vertical resolution of $1 \mathrm{~mm}$ is used. It results from a compromise between maximum resolution along the axis of the crack, and lower resolution along the axis perpendicular to the crack, thus allowing the computing time to be optimized.

Diffuse energy is initiated by the experimentally determined energy spectrum of the 500 $\mathrm{kHz}$ sensor used in [14]. Ultrasonic energy diffusion is simulated by directly applying the general diffusion equation (Eq. 1), using the energy emitted by the source $\left(x_{e x c}, y_{\text {exc }}\right)$, expressed in the form of a Dirichlet condition (Eq. 2). 


$$
\begin{aligned}
& \frac{\partial}{\partial t}\langle E(x, y, t)\rangle+\sigma\langle E(x, y, t)\rangle=D \Delta\langle E(x, y, t)\rangle, \\
& \left\langle E\left(x_{e x c}, y_{e x c}, t\right)\right\rangle=E_{0}(t),
\end{aligned}
$$

Using $E_{i, j}{ }^{(n)}$ to note the energy at point $\left(x_{i}, y_{i}\right)=\left(i \Delta x+x_{0}, j \Delta y+y_{0}\right)$, and at instant $n \Delta t$, the resolution scheme can be written in an explicit temporal form:

$$
\frac{E_{i, j}^{(n+1)}-E_{i, j}^{(n)}}{\Delta t}=-\sigma E_{i, j}^{(n)}+D \frac{E_{i-1, j}^{(n)}-2 E_{i, j}^{(n)}+E_{i+1, j}^{(n)}}{\Delta x^{2}}+D \frac{E_{i, j-1}^{(n)}-2 E_{i, j}^{(n)}+E_{i, j+1}^{(n)}}{\Delta y^{2}},
$$

This explicit form implies that the Courant-Friedrich-Lévy (CFL) condition is respected, i.e. that the temporal interval remains below a certain maximum value, which depends on the spatial interval, such that the information supplied by a given point is correctly transmitted to the adjacent ones. In the case of the present equation, the CFL condition is given by;

$$
\Delta t \leq \frac{\min \left(\Delta x^{2}, \Delta y^{2}\right)}{D}
$$

In the following simulations, this leads to the requirement: $\Delta t<1.710^{-7} \mathrm{~s}$, which is of a similar order to the value of $\Delta t$ needed to account for the highest signal frequency. It is of the order of $700 \mathrm{kHz}$, thus, in this simulation the CFL does not lead to any limitation. In order to optimize the computation time, only a $10 \times 30 \mathrm{~cm}$ portion of the concrete surrounding the crack (Fig. 2) is modeled. The boundary conditions of the model are chosen as: no energy at the concrete-concrete interfaces (in green, Fig. 2), Neumann conditions at the concrete-air interface (shown in red, Fig. 2). The dissipation in concrete is sufficiently great to avoid having to take the absorbent layers onto account. This corresponds to a so-called "Perfectly Matched Layer" (PML), and prevents reflections. Diffusion parameters in Eq.(3) are assumed to be frequency independent: taking a frequency dependence into account would require frequency domain analysis, which would add considerable complexity to the resolution scheme, involving the management of parasitic oscillatory effects arising from the inverse Fourier transform.

This numerical model allows crack parameters such as depth and geometry to be modified. It also allows the real crack morphology to be accounted for, by creating partial contacts along the length of the crack. An opened crack, or a crack with partial opened portions, is produced in the numerical model by imposing the Neumann conditions on the walls of the crack:

$$
\left.\frac{\partial}{\partial n}\langle E(x, y, t)\rangle\right|_{\Gamma}=0
$$

where $\Gamma$ indicates the walls of the crack and $n$ is the normal at a point on this wall. In the simulation, in which vertical cracks are considered, this amounts to writing the expressions for the left and right walls of the crack, respectively as: 


$$
\frac{E_{i-1, j}^{(n)}-E_{i, j}^{(n)}}{\Delta x}=0, \frac{E_{i+1, j}^{(n)}-E_{i, j}^{(n)}}{\Delta x}=0 .
$$

This condition ensures that no energy can flow through the crack. The so-called open crack is defined by its depth (Figs. 3a, 3b). A realistic crack is introduced by the addition of partial contacts (Figs. 3c, 3d). To study their influence on diffuse ultrasound, the number and distribution of these contacts are parameterized. These points allow a part of diffuse energy to be transmitted through the crack as shown in Fig. 4.

In the following section, the model is validated by comparing its predictions with the experimental results presented by Quiviger et al. [14] for the case of open cracks (notches). The influence of contact points along the length of the crack is then studied. A realistic crack morphology is proposed in order to compare the model with experimental results obtained by Quiviger et al. [14] for the case of real cracks.

\section{III.2 Validation of the model}

The validation of the model is performed by comparing its predictions with the experimental results presented by Quiviger et al. [14], for the case of open cracks (notches). In the simulations, the diffusivity and dissipation parameters, $D$ and $\sigma$, are constants. The experimental values from [14] of $D$ and $\sigma$ of concrete without cracks are introduced as inputs in the model. Following the signal processing proposed in [14], the apparent diffusion parameters are determined, in order to compare the numerical results with those found experimentally. They are evaluated through the use of a least squares fit to the solution of the 2D energy diffusion equation from the energy measured by the numerical receiver. Further details about diffusion constant determination are available in [14]. The results of the numerical simulations are shown in Fig. 5 with notches with depths ranging from 1 to $5 \mathrm{~cm}$.

The numerical results show that the variation in these apparent parameters, as a function of the depth of the open crack, lies within the range of uncertainty of the experimental values, with the exception of the dissipation determined for a $2 \mathrm{~cm}$ notch. Also, the ATME values reveal a similar variation, with a difference which could arise from the boundary conditions of the simulation as well as the 2D limitations of the model. These results allow the diffuse energy simulator, as well as the data processing, to be validated.

\section{III.3 Simulation of partial contacts}

In the presence of a closed crack, Quiviger et al. [14] assume that the contact zones between the walls of the crack play a predominant role. The present study focuses on the ability of the proposed simulation to accurately simulate diffuse ultrasound propagation through a realistic $2 \mathrm{D}$ numerical crack. Due to the mesh defined previously and the $2 \mathrm{D}$ nature of the simulation, the crack is assumed to represent the projection of the $3 \mathrm{D}$ crack morphology in the simulation plane. In order to reduce the parametrical study, the contact zones are defined as single mesh points $\left(1 \times 2 \mathrm{~mm}^{2}\right)$ located along a line. In the following, the influence of the number of contact points on the diffusion phenomenon is evaluated. The influence of the position of a single contact point along the crack is determined. Then, the experimental data from real cracks [14], together with the detailed morphology described in the second part of this paper, are used to optimize 
the model. The experimental data shows that the most relevant parameter is the ATME [14]. Thus, the remaining part of the study is restricted to the use of the latter parameter.

\section{III.3.1 Number of contacts}

In order to evaluate the consequences of a variation in the density of contact points on the diffusion properties through a closed crack, a crack is simulated with a total length of $5 \mathrm{~cm}$, including from 1 to 24 contacts, linearly distributed along the crack [Fig. 6(ab)].

The simulations and data processing are carried out according to the same procedures as those described in the foregoing descriptions. The results plotted Fig. 6 show that the ATME decreases as a function of the number of contact points. It is noticeable that the presence of a single contact point located half length of the crack leads to a $5 \mu$ s, i.e. approximately $10 \%$, change in ATME. With increasing contact density, the ATME decreases, approaching the direct trajectory time.

\section{III.3.2 Contact point locations}

The position of a contact point along the crack is the second parameter influencing diffuse energy propagation. Fig. 7 shows the influence of a single contact point located along the length of the crack at a depth ranging from 1 to $50 \mathrm{~mm}$.

The distance between the contact point and the surface has an influence on the propagation of diffuse energy through the crack. ATME increases with the depth of the contact point. Note that for the given crack depth $(5 \mathrm{~cm})$, the different contact point locations lead to an ATME variation of the order of $10 \mu \mathrm{s}$.

\section{III.3.3 Real cracks}

This section deals with the variations in ATME as a function of the depth of the cracks observed experimentally by Quiviger et al. [14]. The cracks were generated using threepoint bending, initiated by a $1 \mathrm{~cm}$ notch at the center of the test specimen. It is also modeled in the following simulations. The simulated cracks, identical to those analyzed experimentally $(1,3$ and $5.5 \mathrm{~cm})$, are represented by a linear distribution of contact points along the length of the crack (Fig. 8). This distribution is in agreement with observations made by Turatsinze et al. [15], described in section 2 . The contact density is the optimization parameter used in the numerical model to match experiments from [14]. An iteration process modifies this contact density, in order to optimize the morphology of the numerical crack with respect to the experimental results. The results are shown in Fig. 9.

It shows that the variations in the simulated and experimental results are in agreement. Into this optimized configuration, the contact density is found to represent $6.5 \%$ of the total length of the crack. It should be noted that the numerical results for the ATME reach a maximum value at a depth of $4 \mathrm{~cm}$, and then fall to a lower value at a depth of $6.5 \mathrm{~cm}$, whereas the experimental results show a continuous increase in ATME. Nevertheless, the numerical variations remain within the range of uncertainty of the experimental results. This difference may be due to the crack description which takes into account a linear contact point distribution along the crack.

\section{Conclusion}

The simulation tool and the numerical description of a crack allow a better understanding of diffuse wave's interactions with a real crack. A realistic numerical 
diffusion model including dissipation is proposed and validated through the use of experimental results from the literature. The simulations reveal the influence of the number of contacts, and their spatial distribution on the measured ATME. A realistic crack morphology is described numerically which makes possible the model to be optimized. It is shown that a linear contact density of $6.5 \%$ can explain the experimental variations in ATME, for cracks varying between 1 and $5.5 \mathrm{~cm}$ in depth. A more complex distribution could have been used but there is a lack of knowledge about crack morphology to validate such a study.

Fig. 1 shows the 3D characteristics of the crack, with varying contact densities between the test specimen's outer surfaces and its center. However it is important to note that Turatsinze et al. produced an image of the crack by slicing the samples. That leads to internal stress release, which can produce openings which are not present in bulk of the samples.

In this study, as regard with the symmetric location of the transducers and the position of the cracks, the 2D simulation is accurate. A $3 \mathrm{D}$ extension of this simulation is currently being evaluated. It will allow to model non-symmetric samples as well as various transducer locations. However, the main issue concerns the knowledge of the crack morphology in the bulk of the material. Non-destructive 3D observation of the morphology of this type of crack is to be considered in future studies, using X-ray microtomography for example. The complementarity of these data should ultimately allow the 3D crack morphology to be imaged in concrete.

The numerical model presented in this paper will also be of interest in future studies dealing with gradual changes in depth. As this simulation method includes the dissipation, it allows to simulate some concrete pathologies such as carbonation by introducing gradual changes of porosity (which mainly drives the dissipation) in the firsts centimeters of concrete.

\section{Acknowledgements}

Authors wish to thank Electricité de France for funding this study. Jean-Paul Balayssac (LMDC, France) is also acknowledged for helpful comments and suggestions about crack morphology in concrete. 


\section{References}

[1] ISO 1920-7:2004, Testing of concrete, Part 7: Non-destructive tests on hardened concrete.

[2] ASTM C597-09, Standard Test Method for Pulse Velocity Through Concrete

[3] Chaix JF, Garnier V, Corneloup G. Ultrasonic wave propagation in heterogeneous solid media: Theoretical analysis and experimental validation. Ultrasonics 2006;44:200210.

[4] Payan C, Garnier V, Moysan J and Johnson PA. Determination of third order elastic constants in a complex solid applying coda wave interferometry. App. Phys. Lett. 2009;94:011904.

[5] Van Hauwaert A., Thimus JF, and Delannay F. Use of Ultrasonics to Follow Crack Growth. Ultrasonics 1998;36:209-217.

[6] Hévin G, Abraham O, Pedersen HA, and Campillo M. Characterization of Surface Cracks with Rayleigh Waves: a Numerical Model. NDT\&E Int 1998;31:289-297.

[7] Liou T., Hsiao C, Cheng CC, and Chang N. Depth Measurement of Notches as Models for Shallow Cracks in Concrete. NDT\&E Int 2009;42(1):69-76.

[8] Aggelis DG, Kordatos EZ, Soulioti DV, Matikas TE. Combined Use of Thermography and Ultrasound for the Characterization of Subsurface Cracks in Concrete. Con. Build. Mat. 2010;24(10):1888-1897.

[9] Anugonda P, Wiehn J and Turner J. Diffusion of ultrasound in concrete. Ultrasonics 2001;39(6):429-435.

[10] Becker J, Jacobs LJ and Qu J. Characterization of Cement-Based Materials Using Diffuse Ultrasound. Journal of Engineering Mechanics 2003;129(12):1478-1484.

[11] Punuraia W, Jarzynskib J, Qub J, Kurtisa KE and Jacobs LJ. Characterization of dissipation losses in cement paste with diffuse ultrasound. Mech. Res. Com. 2007;34(3):289-294.

[12] Deroo F, Kim JY, Qu J, Sabra K, Jacobs LJ. Detection of damage in concrete using diffuse ultrasound. J Acoust Soc Am. 2010;127(6):3315-3318.

[13] Ramamoorthy SK, Kane Y and Turner JA. Ultrasound diffusion for crack depth determination in concrete. J. Acoust. Soc. Am. 2004;115(2):523-529.

[14] Quiviger A, Payan C, Chaix JF, Garnier V, Salin J. Effect of the presence and size of a real macro-crack on diffuse ultrasound in concrete. NDT\&E Int 2012;45(1):128132

[15] Turatsinze A and Bascoul. A. Restrained Crack Widening in Mode I Crack Propagation for Mortar and Concrete. Advanced Cement Based Materials 1996;4(34):77-92.

[16] Seher M, In CW, Kim JY, Kurtis KE, Jacobs LJ. Numerical and Experimental Study of Crack Depth Measurement in Concrete Using Diffuse Ultrasound, Journal of Nondestructive Evaluation 2013;32(1):81-92. 


\section{FIGURE CAPTION}

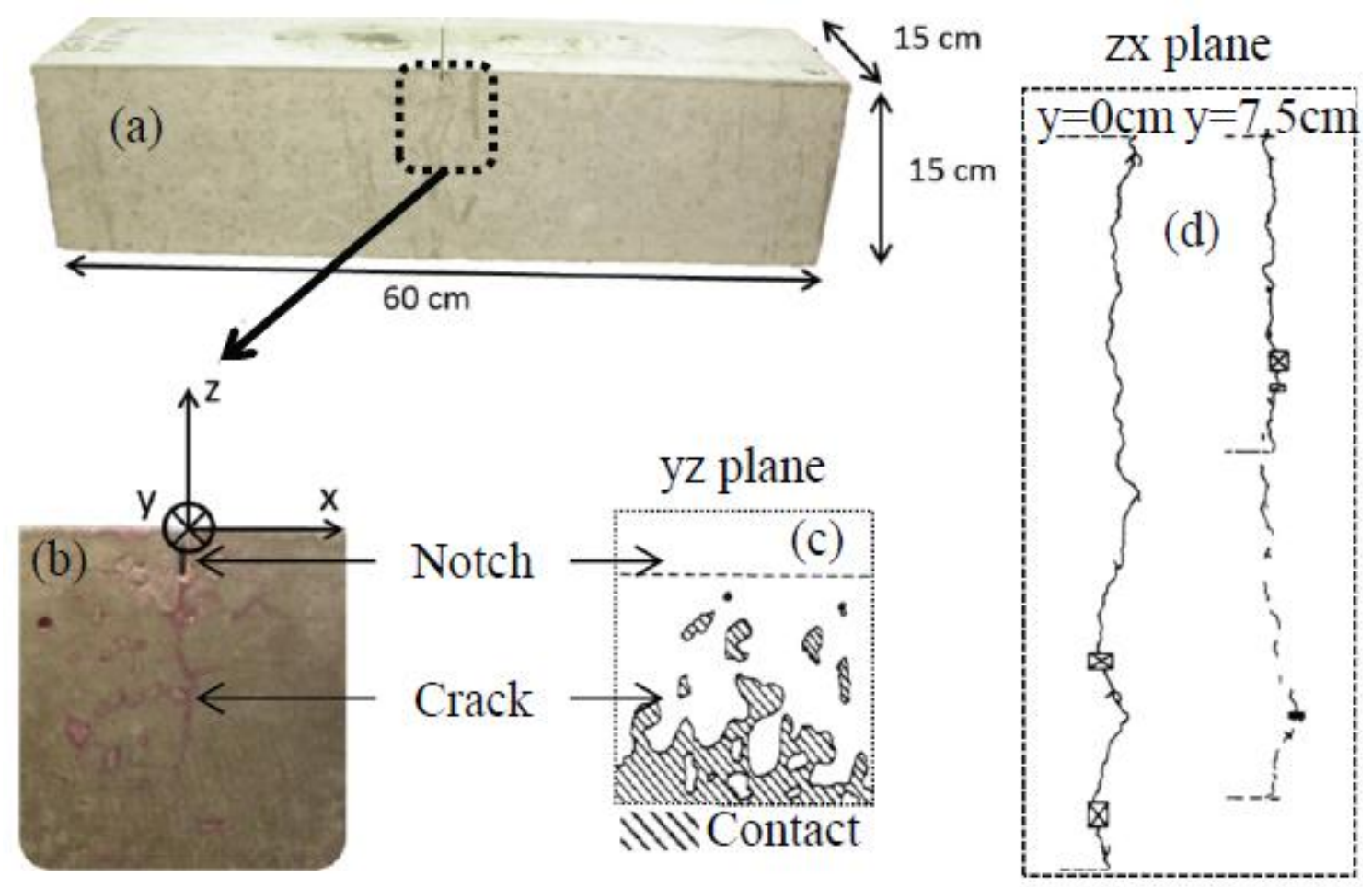

Figure 1: Map of a real crack in concrete: (a) concrete samples used in the present study, (b) zoom on the crack region revealed by dye penetrant inspection at the surface (present study), (c) crack observation from [15] in a longitudinal plane at $\mathrm{y}=0$ (surface) and $y=7.5 \mathrm{~cm}$ (center), (d) transverse observation at $\mathrm{x}=0$ from [15]. 


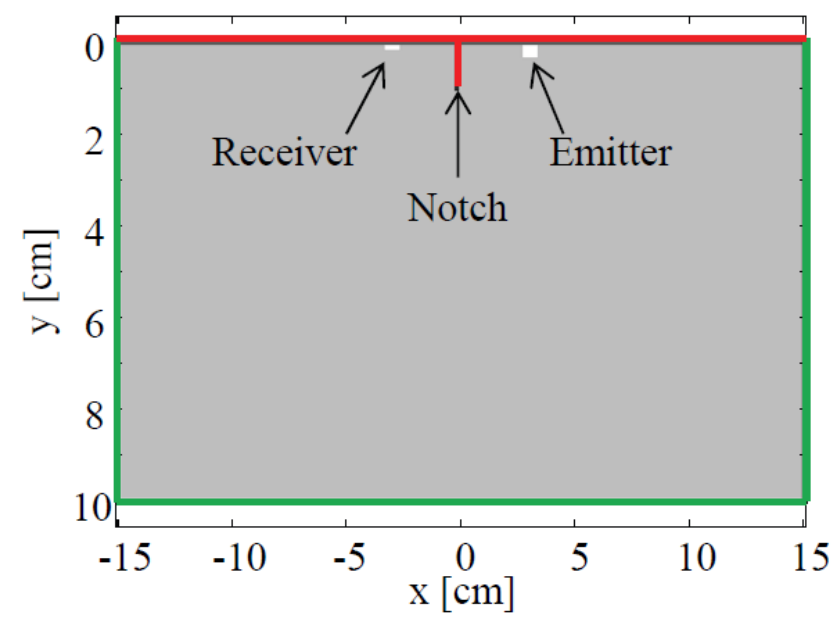

Figure 2: (Color online) Cross-section of the modelled $10 \times 30 \mathrm{~cm}$ concrete specimen. A $(1 \mathrm{~cm})$ notch is positioned at the origin. The positions of the two transducers are shown, on either side (at $x=+3$ and $-3 \mathrm{~cm}$ ) of the notch. No energy boundary condition in green and Neumann boundary condition in red. 

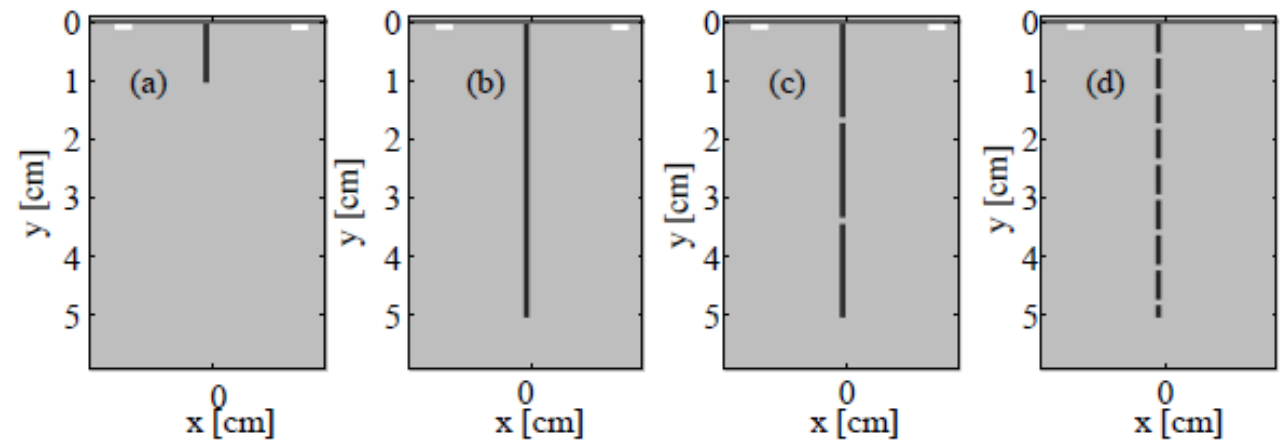

Figure 3: (a-b) Detailed views of the concrete test specimen, showing two examples of an open crack, with depths equal to 1 and $5 \mathrm{~cm}$. (c-d) Detailed views of the concrete test specimen, showing two examples of a $5 \mathrm{~cm}$ deep closed crack, with respectively 2 and 8 points of contact. 


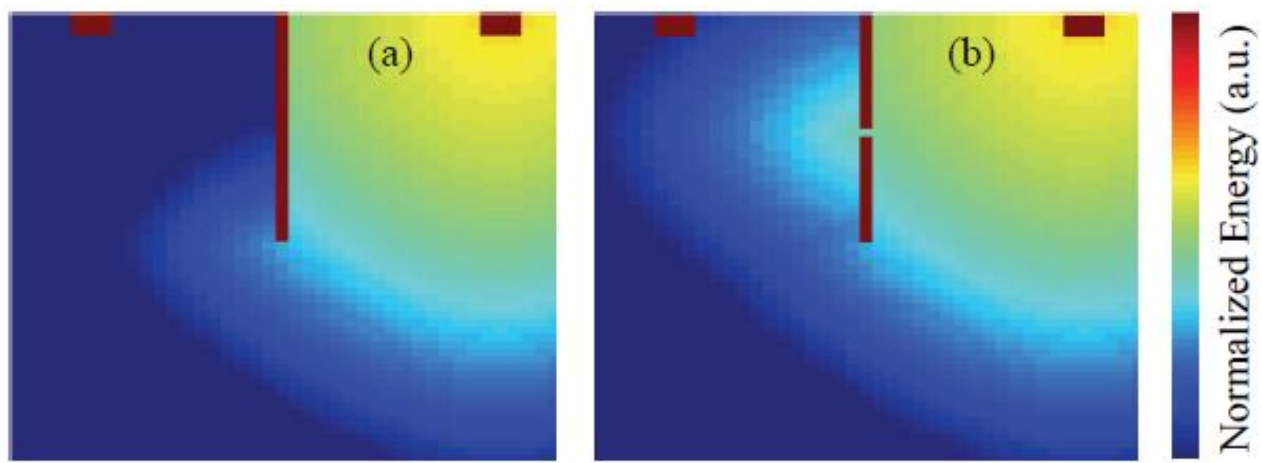

Figure 4: (Color online) Snapshot at the same time of diffuse ultrasound energy with (a) a notched sample and (b) the same one with a single contact point at the notch center. 

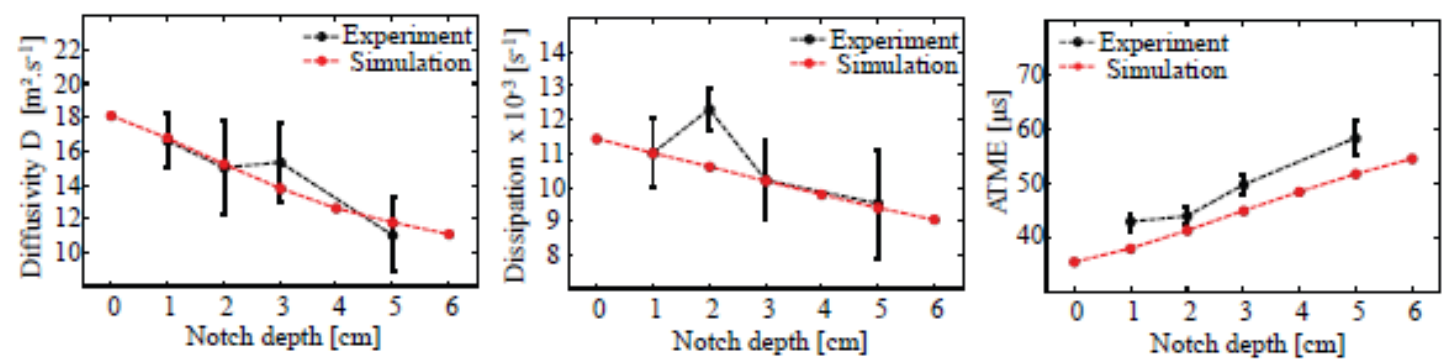

Figure 5: (Color online) Comparison of apparent diffusion parameters derived from the simulation, with those determined experimentally, for a notch of variable length. 

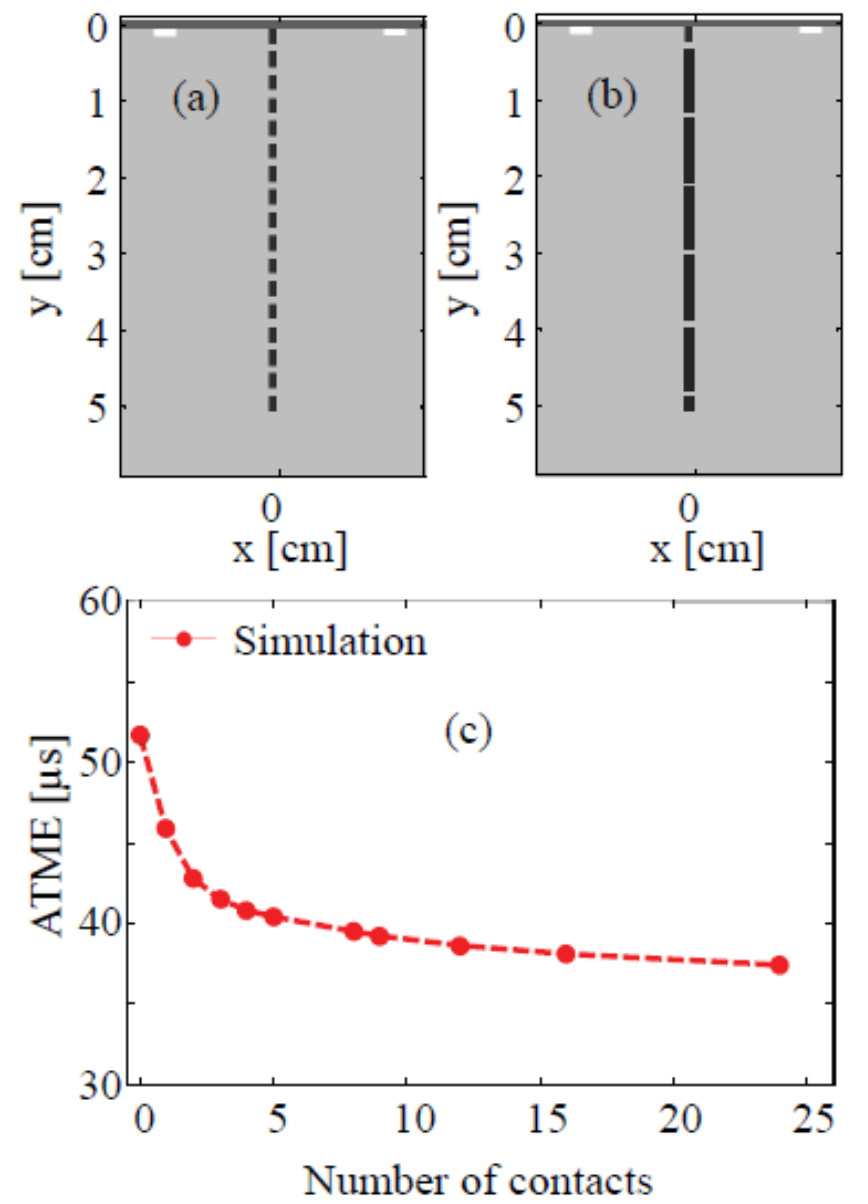

Figure 6: (Color online) (a-b) Example of spatial distribution of contacts along the length of the crack, and (c) variation in ATME as a function of the number of linearly distributed contact points along a total crack length of $5 \mathrm{~cm}$. 

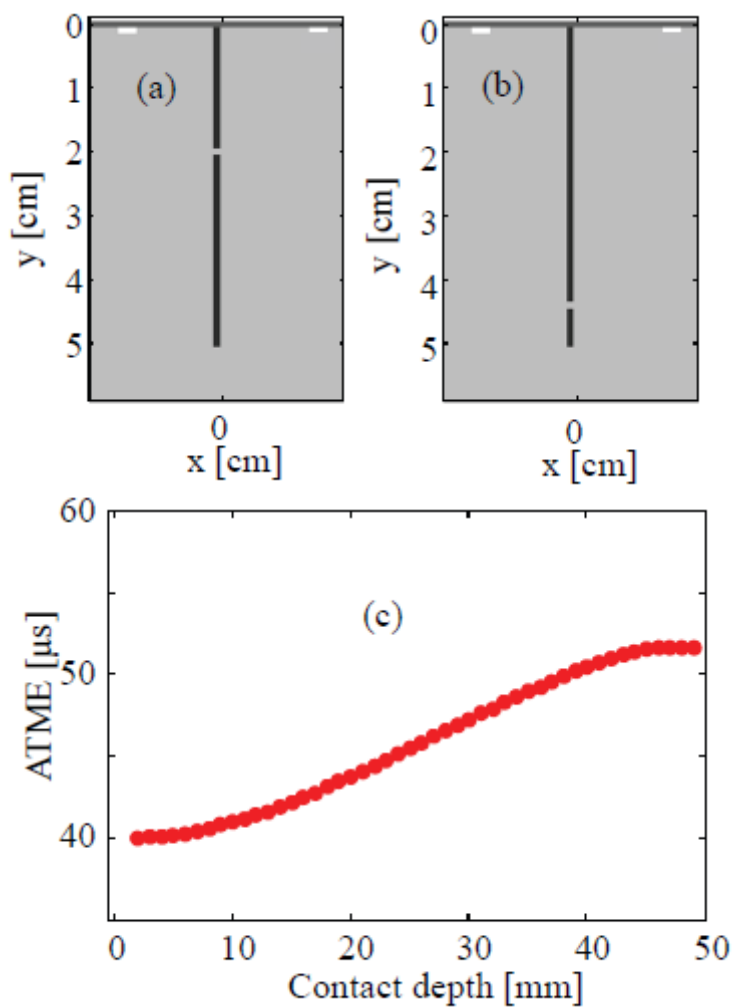

Figure 7: (Color online) (a-b) Example of position of a single contact along a $5 \mathrm{~cm}$ crack. (c) Variation in ATME as a function of the contact position. 

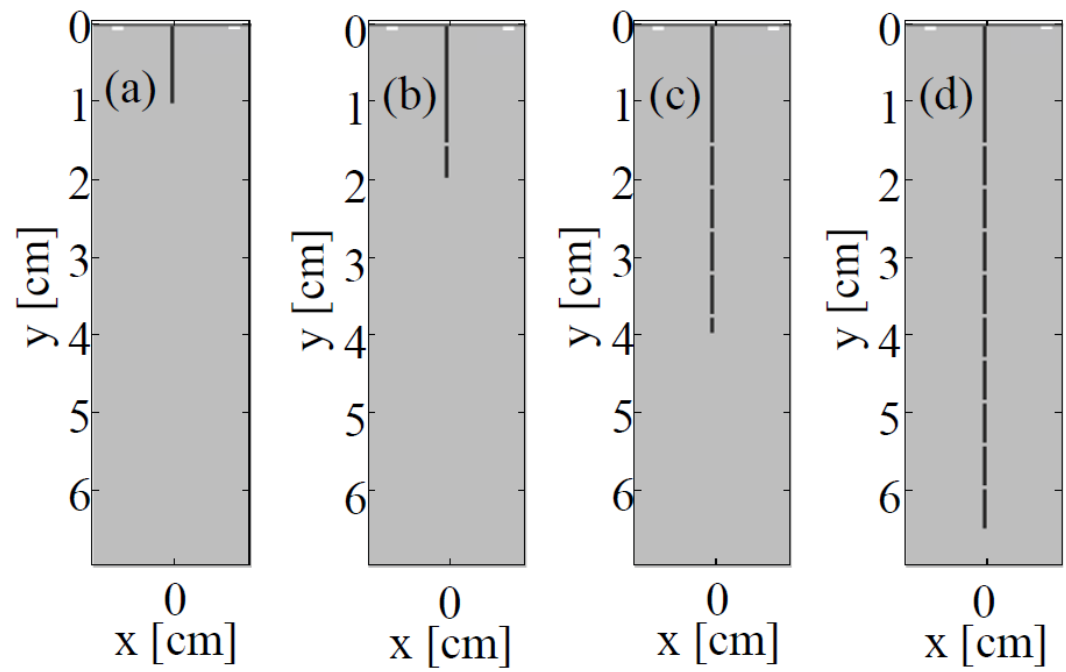

Figure 8: Numerical simulation of the cracks analysed during the experiments. (a) $0 \mathrm{~cm}$, (b) $1 \mathrm{~cm}$, (c) $3 \mathrm{~cm}$ and (d) $5.5 \mathrm{~cm}$. 


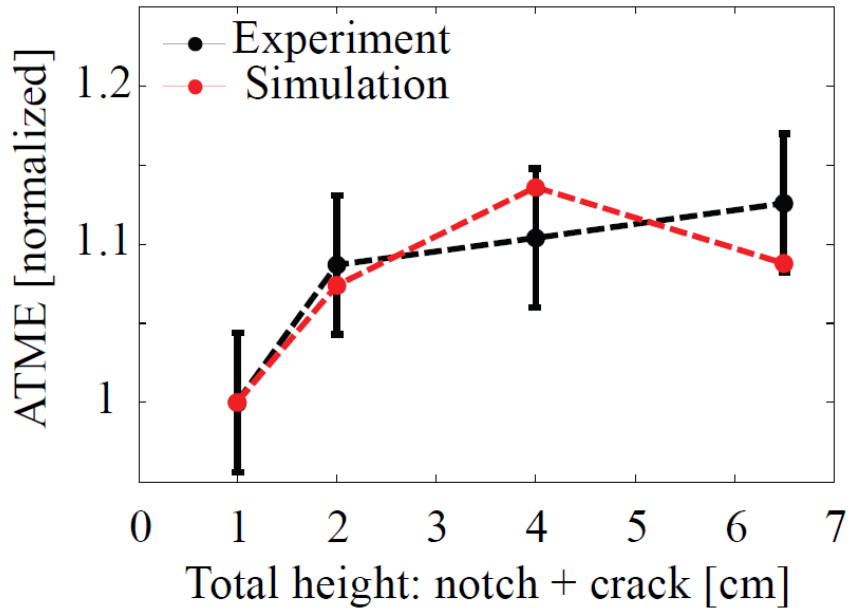

Figure 9: (Color online) Variation of the ATME as a function of the depth of the crack, for an optimized contact density. Comparison with experimental results. 